hep-th/0312274

December 2003

\title{
Ricci flows and infinite dimensional algebras*
}

\author{
Ioannis Bakas \\ Department of Physics, University of Patras \\ GR-26500 Patras, Greece \\ bakas@ajax . physics . upatras.gr
}

\begin{abstract}
The renormalization group equations of two-dimensional sigma models describe geometric deformations of their target space when the world-sheet length changes scale from the ultra-violet to the infra-red. These equations, which are also known in the mathematics literature as Ricci flows, are analyzed for the particular case of two-dimensional target spaces, where they are found to admit a systematic description as Toda system. Their zero curvature formulation is made possible with the aid of a novel infinite dimensional Lie algebra, which has anti-symmetric Cartan kernel and exhibits exponential growth. The general solution is obtained in closed form using Bäcklund transformations, and special examples include the sausage model and the decay process of conical singularities to the plane. Thus, Ricci flows provide a non-linear generalization of the heat equation in two dimensions with the same dissipative properties. Various applications to dynamical problems of string theory are also briefly discussed. Finally, we outline generalizations to higher dimensional target spaces that exhibit sufficient number of Killing symmetries.
\end{abstract}

\footnotetext{
${ }^{*}$ Contribution to the proceedings of the 36th International Symposium Ahrenshoop on the Theory of Elementary Particles, Berlin, August 26-30, 2003; to be published in Fortschritte der Physik.
} 
The renormalization group properties of two-dimensional sigma models were extensively studied in the past, and it was found that their beta function $\beta\left(g^{2}\right)$ is not always zero (see, for instance, [1]). In general, the coupling constant runs by changing the length scale of the world-sheet and the theory breaks conformal invariance in the quantum regime. This general framework has two applications in string theory. First, it is interesting to find fixed points of the renormalization group flow, as they provide conformal field theory blocks for building string vacua. Second, it is also interesting to study non-conformal backgrounds by solving the beta function equations and try to develop an off-shell formulation for addressing the problem of vacuum selection in string theory. Our work is mostly concerned with the second problem, as we report on a new algebraic method for the integration of the renormalization group equations that appear to lowest order in perturbation theory. Further details can be found in a recent publication, [2].

We set us the notation by considering two-dimensional sigma models with classical action

$$
S=\frac{1}{4 \pi \alpha^{\prime}} \int d^{2} w \sqrt{h} h^{i j}\left(\partial_{i} X^{\mu}\right)\left(\partial_{j} X^{\nu}\right) G_{\mu \nu},
$$

where $\left\{X^{\mu} ; \mu=1,2, \cdots, n\right\}$ are coordinates in target space with metric $G_{\mu \nu}(X)$. Let $\Lambda^{-1}$ be the renormalization scale parameter on the world-sheet and $R_{\mu \nu}$ the Ricci curvature tensor of the target space metric. Then, the renormalization group equations are

$$
\Lambda^{-1} \frac{\partial}{\partial \Lambda^{-1}} G_{\mu \nu}=-\beta\left(G_{\mu \nu}\right)=-R_{\mu \nu}+\cdots
$$

in appropriate units, $2 \pi \alpha^{\prime}=1$, and dots denote higher order curvature terms that arise at two loops or higher in perturbation theory, [3]. We will only consider the lowest order terms, which provide a good approximation to the metric beta functions when the curvature is rather weak, and define the logarithmic length scale $t=\log \Lambda^{-1}$ as the renormalization group time; in this parameterization, the ultra-violet limit corresponds to $t \rightarrow-\infty$ and the infra-red limit to $t \rightarrow+\infty$. Thus, we consider the equations

$$
\frac{\partial}{\partial t} G_{\mu \nu}=-R_{\mu \nu},
$$

which define a dynamical system in superspace that consists of all possible metrics.

These equations describe changes of the target space geometry that are induced by changes of the world-sheet logarithmic scale, but they are very difficult to solve in all generality. The same equations also arose independently in the mathematics literature, where they became known as Ricci flows, and they have turned into a major tool for addressing a variety of open problems in geometry in diverse dimensions; for a recent account of the main mathematical results see, for instance, [4], and references therein. We will be able to solve these equations for two-dimensional target spaces by casting them into zero curvature form and then integrate them in closed form. As byproduct, we reveal the role of a novel infinite dimensional Lie algebra in this dynamical problem, which arises in the zero curvature formulation and it could be of more general value. Some special trajectories will also be briefly reviewed in order to confront the algebraic 
construction of the general solution with the geometric deformations that are involved. We also note for completeness that the renormalization group equations (3) admit a more general description in the form

$$
\frac{\partial}{\partial t} G_{\mu \nu}=-R_{\mu \nu}+\nabla_{\mu} \xi_{\nu}+\nabla_{\nu} \xi_{\mu}
$$

which incorporates the effect of all possible field redefinitions $\delta X_{\mu}=-\xi_{\mu}$ associated with changes of coordinates along the flow. Some solutions assume a simpler form when they are written in different frames.

Let us now restrict attention to two-dimensional target spaces in the conformally flat frame

$$
d s_{\mathrm{t}}^{2}=2 e^{\Phi\left(z_{+}, z_{-} ; t\right)} d z_{+} d z_{-}=\frac{1}{2} e^{\Phi(X, Y ; t)}\left(d X^{2}+d Y^{2}\right)
$$

using Cartesian coordinates $X, Y$ or the complex conjugate variables $2 z_{ \pm}=Y \pm i X$. Then, since the non-vanishing component of the Ricci curvature tensor equal to

$$
R_{+-}=-\partial_{+}\left(e^{-\Phi} \partial_{-} e^{\Phi}\right)=-\nabla^{2} \Phi
$$

we find that the renormalization group flows (3) reduce in this frame to the following non-linear differential equation for $\Phi(X, Y ; t)$,

$$
\frac{\partial}{\partial t} e^{\Phi(X, Y ; t)}=\nabla^{2} \Phi(X, Y ; t)
$$

This equation provides a non-linear generalization of the heat equation on the twodimensional plane,

$$
\frac{\partial}{\partial t} \Theta(X, Y ; t)=\nabla^{2} \Theta(X, Y ; t)
$$

which approximates $(7)$ when $\Phi(X, Y ; t) \simeq \Theta(X, Y ; t)$ becomes very small. Such linearization typically arises in the infra-red region of the renormalization group flow, $t \rightarrow+\infty$, where the target space metric becomes approximately flat and the weak field limit

$$
G_{\mu \nu} \simeq \delta_{\mu \nu}+h_{\mu \nu} \quad \text { with } \quad h_{\mu \nu}<<1 \text { and } \partial h_{\mu \nu}<<1
$$

comes into play. The heat equation exhibits dissipative behavior in time, $t$, which can be seen by considering the fundamental solution

$$
\Theta(X, Y ; t)=\frac{1}{4 \pi t} e^{-\left(X^{2}+Y^{2}\right) / 4 t}
$$

that describes a Gaussian pulse with height $1 / t$ and width $\sqrt{t}$. It starts as singular function at the initial time $t=0$, where the Gaussian becomes delta function, and it dissolves after infinitely long time by spreading the initial singularity all over the plane. However, since the weak field approximation (9) is not valid for finite values of $t$, but only asymptotically as $t \rightarrow+\infty$, one may wonder whether the characteristic property of the heat equation to dissipate singularities is also a property of the non-linear equation (7). As we will see later, the decay process of a conical singularity, which describes the 
transition of a flat cone $C / Z_{n}$ to the plane, provides an analogue of the fundamental solution of the heat equation to the non-linear system (7).

The main equation (7) can be conveniently written in the form

$$
\nabla^{2} \Phi(X, Y ; t)=\int d t^{\prime} K\left(t, t^{\prime}\right) e^{\Phi\left(X, Y ; t^{\prime}\right)}
$$

where $K\left(t, t^{\prime}\right)$ is the kernel

$$
K\left(t, t^{\prime}\right)=\frac{\partial}{\partial t} \delta\left(t, t^{\prime}\right)
$$

which is anti-symmetric with respect to its arguments $t$ and $t^{\prime}$. This formulation proves advantageous for casting the Ricci flow into Toda form for appropriate choice of the Lie algebra. Recall at this point that Toda equations describe integrable interactions for a collection of two-dimensional fields $\left\{\phi_{i}(X, Y)\right\}$, which are labeled by the simple roots of a given Lie algebra and they are coupled via the Cartan matrix $K_{i j}$ as follows,

$$
\nabla^{2} \phi_{i}(X, Y)=\sum_{j} K_{i j} e^{\phi_{j}(X, Y)}
$$

The indices $i, j$ are typically discrete, but there are also generalizations to continuous variables for some infinite dimensional Lie algebras, which are obtained by replacing the collection of two-dimensional Toda fields $\left\{\phi_{i}\right\}$ with a "master" field $\Phi(X, Y ; t)$, the Cartan matrix $K_{i j}$ with a Cartan kernel $K\left(t, t^{\prime}\right)$, and the summation over $j$ with an integral over $t^{\prime}$. In this case, $t$ is interpreted as Dynkin parameter on the root system of a continual Lie algebra whose basic commutation relations are defined by the corresponding Cartan kernel, which depends on continuous variables rather than discrete labels. The Ricci flows in two dimensions fit precisely this algebraic framework provided that $K\left(t, t^{\prime}\right)$ is given by (12) with $t$ being the logarithm of the world-sheet length scale of sigma models. Its anti-symmetry is inherited from the first order derivatives that describe the evolution in $t$.

The notion of continual Lie algebras arises naturally by considering a system of Cartan-Weyl generators $\left\{H(t), X^{ \pm}(t)\right\}$ that depend on a continuous variable $t$, so that the commutation relations of the local part of the algebra assume the general form, [5]

$$
\begin{aligned}
& {\left[X^{+}(t), X^{-}\left(t^{\prime}\right)\right]=\delta\left(t-t^{\prime}\right) H\left(t^{\prime}\right), \quad\left[H(t), H\left(t^{\prime}\right)\right]=0} \\
& {\left[H(t), X^{ \pm}\left(t^{\prime}\right)\right]= \pm K\left(t, t^{\prime}\right) X^{ \pm}\left(t^{\prime}\right) .}
\end{aligned}
$$

Equivalently, using the smeared form of the generators $\left\{H(\varphi), X^{ \pm}(\varphi)\right\}$ with respect to arbitrary function $\varphi(t)$, where

$$
A(\varphi)=\int d t \varphi(t) A(t)
$$

the commutation relations above assume the form

$$
\begin{aligned}
& {\left[X^{+}(\varphi), X^{-}(\psi)\right]=H(\varphi \psi), \quad[H(\varphi), H(\psi)]=0,} \\
& {\left[H(\varphi), X^{ \pm}(\psi)\right]= \pm X^{ \pm}(K(\varphi, \psi)) .}
\end{aligned}
$$


Here, we restrict attention to bilinear maps $K(\varphi, \psi)=(K \varphi) \cdot \psi$, which are described by a much simpler linear map $K$, so that there is always consistency with the Jacobi identities. The algebra that results in this case is denoted by $\mathcal{G}(K, 1)$ and it is $Z$-graded in the sense that

$$
\mathcal{G}(K, 1)=\oplus_{n \in Z} \mathcal{G}_{n}
$$

The local part corresponds to $\mathcal{G}_{-1} \oplus \mathcal{G}_{0} \oplus \mathcal{G}+1$, whereas all other elements are obtained, as usual, by taking successive commutators so that $\mathcal{G}_{n}=\left[\mathcal{G}_{n-1}, \mathcal{G}_{+1}\right]$ for $n>0$ and $\mathcal{G}_{n}=\left[\mathcal{G}_{n+1}, \mathcal{G}_{-1}\right]$ for $n<0$.

The Ricci flows admit a zero curvature formulation as Toda theory for the special continual Lie algebra with $K=d / d t$, [2]. It can be easily seen by considering

$$
\left[\partial_{+}+A_{+}\left(z_{+}, z_{-}\right), \partial_{-}+A_{-}\left(z_{+}, z_{-}\right)\right]=0
$$

where the gauge connections $A_{ \pm}$take values in the local part of the algebra $\mathcal{G}_{0} \oplus \mathcal{G}_{ \pm 1}$, respectively, with

$$
A_{ \pm}\left(z_{+}, z_{-}\right)=H\left(u_{ \pm}\right)+\lambda X^{ \pm}\left(f_{ \pm}\right)
$$

that depend on some arbitrary functions $u_{ \pm}$and $f_{ \pm}$of $z_{+}, z_{-}$and $t$. Then, using the basic commutation relations of the algebra $\mathcal{G}(d / d t ; 1)$ we obtain the following system of equations

$$
\begin{aligned}
& \frac{\partial u_{+}}{\partial t}=-\partial_{+}\left(\log f_{-}\right), \quad \frac{\partial u_{-}}{\partial t}=\partial_{-}\left(\log f_{+}\right), \\
& \partial_{+} u_{-}-\partial_{-} u_{+}+\lambda^{2} f_{+} f_{-}=0,
\end{aligned}
$$

which can be simplified by eliminating $u_{ \pm}$. Then, the zero curvature condition yields

$$
\partial_{+} \partial_{-} \Phi\left(z_{+}, z_{-} ; t\right)=-\lambda^{2} \frac{\partial}{\partial t} e^{\Phi\left(z_{+}, z_{-} ; t\right)}, \quad \text { with } \quad \Phi=\log \left(f_{+} f_{-}\right),
$$

which is the Ricci flow equation (7) in conformally flat coordinates $z_{+}, z_{-}$with $\lambda^{2}=-1$. Equivalently, using the gauge invariance of the zero curvature condition, we may choose $u_{-}=0, f_{+}=1$ from the very beginning, and set $f_{-}=\exp \Phi, u_{+}=\Psi$, where $\partial_{+} \Phi=-\partial_{t} \Psi$. Thus, the renormalization group equations are integrable in target space, while $t$ enters into the definition of the infinite dimensional Lie algebra that describes them as continual Toda system.

According to the general theory of Toda field equations, the general solution can be obtained in closed form, using Bäcklund transformations, and it is parametrized by a system of arbitrary free fields. The group theoretical method that allows for their integration is based on the existence of a highest weight state $\mid t>$, so that

$$
X^{+}\left(t^{\prime}\right)|t>=0,<t| X^{-}\left(t^{\prime}\right)=0, H\left(t^{\prime}\right)\left|t>=\delta\left(t-t^{\prime}\right)\right| t>
$$

with $\langle t \mid t\rangle=1$. This is formally defined for continual Lie algebras, in analogy with the representations of finite dimensional simple Lie algebras. Then, for the case at hand, the general solution assumes the form, [2]

$$
\Phi\left(z_{+}, z_{-} ; t\right)=\Phi_{0}\left(z_{+}, z_{-} ; t\right)+\partial_{t}\left(\log <t\left|M_{+}^{-1}\left(z_{+} ; t\right) M_{-}\left(z_{-} ; t\right)\right| t>\right),
$$


where $M_{ \pm}$are path-ordered exponentials

$$
M_{ \pm}\left(z_{ \pm} ; t\right)=\mathcal{P} \exp \left(i \int^{z_{ \pm}} d z_{ \pm}^{\prime} \int^{t} d t^{\prime} e^{f^{ \pm}\left(z_{ \pm}^{\prime} ; t^{\prime}\right)} X^{ \pm}\left(t^{\prime}\right)\right)
$$

and $\Phi_{0}\left(z_{+}, z_{-} ; t\right)=f^{+}\left(z_{+} ; t\right)+f^{-}\left(z_{-} ; t\right)$ is a one-parameter family of free fields in two dimensions, i.e., $\partial_{+} \partial_{-} \Phi_{0}\left(z_{+}, z_{-} ; t\right)=0$, which depend on the continuous index $t$.

The solution can be written in formal power series around the free field configuration by expanding the path-ordered exponentials, so that

$$
\begin{gathered}
<t\left|M_{+}^{-1} M_{-}\right| t>=1+\sum_{m=1}^{\infty} \int^{z_{+}} d z_{1}^{+} \cdots \int^{z_{m-1}^{+}} d z_{m}^{+} \int^{z_{-}} d z_{1}^{-} \cdots \int^{z_{m-1}^{-}} d z_{m}^{-} \times \\
\times \int \prod_{i=1}^{m} d t_{i} \int \prod_{i=1}^{m} d t_{i}^{\prime} \exp f^{+}\left(z_{i}^{+} ; t_{i}\right) \exp f^{-}\left(z_{i}^{-} ; t_{i}^{\prime}\right) D_{t}^{\left\{t_{1}, \cdots, t_{m} ; t_{1}^{\prime}, \cdots, t_{m}^{\prime}\right\}} .
\end{gathered}
$$

Here, the matrix elements

$$
D_{t}^{\left\{t_{1}, t_{2}, \cdots, t_{m} ; t_{1}^{\prime}, t_{2}^{\prime}, \cdots, t_{m}^{\prime}\right\}}=<t\left|X^{+}\left(t_{1}\right) X^{+}\left(t_{2}\right) \cdots X^{+}\left(t_{m}\right) X^{-}\left(t_{m}^{\prime}\right) \cdots X^{-}\left(t_{2}^{\prime}\right) X^{-}\left(t_{1}^{\prime}\right)\right| t>
$$

can be evaluated one by one, using the basic commutation relations of the algebra $\mathcal{G}(d / d t ; 1)$, and their integration against the free field $f^{ \pm}$yields all terms in the free field expansion of the Toda field configuration $\Phi\left(z_{+}, z_{-} ; t\right)$. The details are rather cumbersome, since the generic term of the expansion can not be written in closed form, but for special configurations that correspond to free fields

$$
\Phi_{0}\left(z_{+}, z_{-} ; t\right)=c \cdot\left(z_{+}+z_{-}\right)+d(t) \equiv c Y+d(t)
$$

the result is more manageable. Such configurations are used to parametrize solutions of the Toda field equation with an isometry, i.e., independent of $X$, and they give rise to the following perturbative expansion, [2]

$$
\Phi=\Phi_{0}+\frac{1}{c^{2}} \partial_{t} e^{\Phi_{0}}+\frac{1}{4 c^{4}} \partial_{t}\left(e^{\Phi_{0}} \partial_{t} e^{\Phi_{0}}\right)+\frac{1}{36 c^{6}} \partial_{t}\left(3 e^{\Phi_{0}}\left(\partial_{t} e^{\Phi_{0}}\right)^{2}+e^{2 \Phi_{0}} \partial_{t}^{2} e^{\Phi_{0}}\right)+\cdots
$$

which is valid around $\exp \Phi \simeq 0$, i.e., for $\Phi_{0} \rightarrow-\infty$.

The infinite dimensional algebra $\mathcal{G}(d / d t ; 1)$ has exponential growth, which is seen by attempting to construct the independent elements that parametrize the subspaces $\mathcal{G}_{n}$ beyond the local part $n=0, \pm 1$. It can be shown by induction that if $\mathcal{G}_{ \pm n}$ is spanned by $d_{n}$ independent elements $X_{ \pm n}^{(1)}, \cdots, X_{ \pm n}^{\left(d_{n}\right)}$, the subspace $\mathcal{G}_{ \pm(n+1)}=\left[\mathcal{G}_{ \pm 1}, \mathcal{G}_{ \pm n}\right]$ will be spanned by the following $2 d_{n}$ independent elements, [5]

$$
\begin{aligned}
& X_{ \pm(n+1)}^{(s)}(\varphi)=\alpha_{n}^{(s)}\left[X_{ \pm 1}(1), X_{ \pm n}^{(s)}(\varphi)\right]-\left[X_{ \pm 1}(\varphi), X_{ \pm n}^{(s)}(1)\right] ; \quad 1 \leq s \leq d_{n} \\
& X_{ \pm(n+1)}^{(s)}(\varphi)=\beta_{n}^{(s)}\left[X_{ \pm 1}(1), X_{ \pm n}^{(s)}(\varphi)\right]+\left[X_{ \pm 1}(\varphi), X_{ \pm n}^{(s)}(1)\right] ; \quad d_{n}+1 \leq s \leq d_{n}
\end{aligned}
$$

for all $n \geq 2$ and for appropriately chosen constants $\alpha_{n}^{(s)}$ and $\beta_{n}^{(s)}$. This formula does not apply for $n=1$, since the two different series of elements are linearly dependent and 
each $\mathcal{G}_{ \pm 2}$ has only one generator. Thus, the dimension of the subspaces $\mathcal{G}_{ \pm n}$ (relative to the dimension of $\mathcal{G}_{0}$, which is taken to be 1) turns out to be

$$
d_{0}=d_{ \pm 1}=1, \quad d_{n}=2^{n-2} \quad \text { for } \quad n \geq 2
$$

and $\mathcal{G}(d / d t ; 1)$ exhibits exponential growth. This makes it rather exotic and also difficult to study in great detail. The complete system of commutation relations is not known in closed form, but fortunately we only need the local part $\mathcal{G}_{-1} \oplus \mathcal{G}_{0} \oplus \mathcal{G}_{+1}$ to write down the Ricci flows in Toda form. It should be noted, however, that generalized systems of Toda field equations can also be written down in zero curvature form $\left[\partial_{+}+A_{+}, \partial_{-}+A_{-}\right]=0$ for appropriately chosen gauge connections $A_{ \pm} \in \mathcal{G}_{0} \oplus \mathcal{G}_{ \pm 1} \oplus \cdots \oplus \mathcal{G}_{ \pm N}$ for all $N$. It is natural to expect that such equations will be associated to the beta functions of higher spin fields beyond the renormalization group equation of the target space metric.

Returning back to the geometry of Ricci flows, we present some special solutions that have received a lot of attention in recent years. The first describes axially symmetric deformations of the round sphere, which look like a sausage that becomes infinitely long in the ultra-violet region, [6]; the flow terminates at a finite scale, say $t=0$, where the configuration collapses to a point and the lowest order approximation to the beta function equations is not valid anymore. The second describes the decay process of a conical singularity by considering an orbifold configuration $C / Z_{n}$ at some initial time, say $t=0$, which then flows to flat space in the infra-red region, [7, 8]. Both examples are tractable and they can be derived by much simpler integration methods, because they correspond to mini-superspace approximation of the Ricci flow equations imposed by axial symmetry. Other special solutions are also known in the literature, [2], and they all fit into the general algebraic scheme that was presented above.

(i) Sausage model: In this case, the conformal factor of the metric assumes the simple form

$$
e^{\Phi(Y ; t)}=\frac{2}{a(t)+b(t) \cosh 2 Y},
$$

where $0 \leq X \leq 2 \pi$ and $-\infty<Y<+\infty$. Then, the flows (3) reduce to the first order system of differential equations

$$
a^{\prime}(t)=2 b^{2}(t), \quad b^{\prime}(t)=2 a(t) b(t)
$$

which are easily solved in the physical region $a(t) \geq b(t) \geq 0$ as follows,

$$
a(t)=\gamma \operatorname{coth}(-2 \gamma t), \quad b(t)=\frac{\gamma}{\sinh (-2 \gamma t)},
$$

with $\gamma$ being an arbitrary non-negative constant and $t$ running from $-\infty$ (ultra-violet) to 0 (big crunch). This special solution admits a free field representation, in the context of equation (28), provided that

$$
\Phi_{0}(Y ; t)=-2 Y+\log \left(\frac{4}{\gamma} \sinh (-2 \gamma t)\right) .
$$


It can also be written in proper coordinates, where it becomes simpler to visualize as sausage,

$$
d s_{\mathrm{t}}^{2}=\frac{k}{\gamma}\left(d \tilde{Y}^{2}+\operatorname{sn}^{2}(\tilde{Y}+K(k) ; k) d X^{2}\right),
$$

by introducing change of coordinates $\tilde{Y}=F(\psi ; k)$ given by the incomplete elliptic integral of the first kind with free parameter $\sin \psi=\tanh Y$ and modulus $k=\tanh (-\gamma t)$. In this frame, however, one has to introduce a compensating vector field $\xi_{\mu}=\partial_{\mu} \tilde{\Phi}$ with

$$
\tilde{\Phi}(\tilde{Y})=\log \Theta(\tilde{Y}+K(k))+\frac{1}{2}\left(\frac{E(k)}{K(k)}-\frac{1}{2} k^{\prime 2}\left(1+\frac{1}{\gamma}\right)\right) \tilde{Y}^{2}
$$

following equation (4). All formulae are written here in terms of the standard Jacobi elliptic and theta functions. As for the solution, it can also be viewed as bound state of two Euclidean two-dimensional black holes which are glued together in the asymptotic region and they evolve by "eating" each other until they reach a singularity at $t=0$.

(ii) Decay of cone: This process is better described in a frame where the $t$ dependence factorizes linearly, as

$$
d s_{\mathrm{t}}^{2}=t\left(f^{2}(r) d r^{2}+r^{2} d \phi^{2}\right), \quad \xi_{r}=\frac{1}{2} r f(r), \quad \xi_{\phi}=0,
$$

where $0 \leq \phi \leq 2 \pi / n$ and the vector field $\xi_{\mu}$ accounts for the formulation of the solution in a non-conformally flat system. Then, the renormalization group equations (4) result into a simple differential equation for $f(r)$ with solution

$$
\left(\frac{1}{f(r)}-1\right) \exp \left(\frac{1}{f(r)}-1\right)=(n-1) \exp \left(n-1-\frac{r^{2}}{2}\right)
$$

that interpolates smoothly between $f=1 / n$ at $r=0$ and $f=1$ at $r=\infty$. Equivalently, introducing the $t$-dependent change of coordinates $\tilde{r}=r \sqrt{t}$, we find that when $\tilde{r}$ is held fixed the limit $t \rightarrow 0$ corresponds to $f \rightarrow 1$ and $t \rightarrow+\infty$ corresponds to $f \rightarrow 1 / n$. Thus, the geometry describes the transition from a cone $C / Z_{n}$, which has opening angle $2 \pi / n$ at the initial time, to the plane which is only reached in the infra-red limit. The solution looks more complicated when formulated in a conformally flat frame, but it can be worked out in detail and compared with the general solution of the Toda field equation for appropriate choice of free field configuration, [2].

The decay process of a conical singularity demonstrates by example the dissipative nature of the Ricci flows, which are capable to spread an initial curvature singularity all over space after infinitely long time; as such, it can be viewed as non-linear generalization of the fundamental Gaussian solution of the heat equation. This process, which is described in the gravitational regime, has several applications in closed string theory while studying the general problem of tachyon condensation. For instance, we may consider ten-dimensional string vacua of the form

$$
\mathcal{C}=\mathrm{CFT}_{2}+R^{7,1},
$$


where the conformal block $\mathrm{CFT}_{2}$ corresponds to the orbifold field theory $C / Z_{n}$. This vacuum is unstable because there are tachyonic states in the twisted sector of the orbifold, which are localized at the tip of the cone, and they induce transitions to more stable vacua by reducing the order of the singularity; the singularity is completely resolved by the decay of the cone to the plane, as given by the above geometric transition in renormalization group time. More generally, tachyon condensation is quite important for addressing the problem of vacuum selection in string theory by dynamical methods. Ricci flows offer a good starting point in this direction.

The deformations of two-dimensional geometries, which are induced by Ricci flows, could be interpreted as time dependent gravitational solutions in $2+1$ dimensions, which are on-shell. It should be noted, however, that the correspondence with dynamical processes in real time has not been made precise in all generality to this day, although special examples that exhibit the same qualitative behavior with the renormalization group flows are known in the literature, [9]. It is expected that a similar dynamical interpretation will be possible in any number of dimensions, and could be used either way. This formulation is quite important for addressing a variety of physically interesting dynamical problems, such as gravitational collapse, in terms of a more fundamental standpoint provided by world-sheet methods. There are critical phenomena associated to gravitational collapse (for a review see, for instance, [10]), which could be better understood in terms of renormalization group flows of $d$-dimensional geometries rather than using dynamical processes in $(d+1)$-dimensional space-time. In this context, it will also be interesting to revisit Zamolodchikov's $c$-theorem, [11], and its effective field theory description as entropy of the Ricci flows, following the recent work of Perelman, [12].

Another interesting problem is the generalization of our algebraic framework to higher dimensional target spaces. The formulation of Ricci flows as zero curvature condition is rather special to two dimensions, but it can be extended to higher dimensional geometries with sufficient number of Killing isometries, so that the metric is effectively described by functions of two coordinates. For example, in three dimensions, we may consider the action of Ricci flows taking place in the restricted class of metrics with Killing coordinate $w$,

$$
d s_{\mathrm{t}}^{2}=V\left(z_{+}, z_{-} ; t\right) d w^{2}+2 e^{\Phi\left(z_{+}, z_{-} ; t\right)} d z_{+} d z_{-},
$$

and try to describe the evolution equations for the functions $V$ and $\Phi$ in a similar way. In this context, the decay of conical geometries might admit a nice interpretation in terms of matter sources coupled to gravity. The systematic extension of our work to all these cases remains open as there are several two-dimensional fields, and not just $\Phi$, which come into play. We hope to be able to report elsewhere whether they all fit in a generalized Toda system, as for two-dimensional geometries.

In summary, the Ricci flows provide a meeting point for many recent developments in physics and mathematics, which could be used further for the benefit of both subjects. Our main contribution in this area is the algebraic formulation of two-dimensional Ricci flows as Toda system, which in turn points to a new class of infinite dimensional algebras that are relevant for addressing dynamical problems. 


\section{Acknowledgments}

This work was supported in part by the European Research and Training Networks "Superstring Theory" (HPRN-CT-2000-00122) and "Quantum Structure of Space-time" (HPRN-CT-2000-00131), as well as the Greek State Foundation Award "Quantum Fields and Strings" (IKYDA-2001-22) and NATO Collaborative Linkage Grant "Algebraic and Geometric Aspects of Conformal Field Theories and Superstrings" (PST.CLG.978785). I also thank the organizers of the meeting for their kind invitation to present the results of my work in a pleasant and stimulating atmosphere.

\section{References}

[1] A.M. Polyakov, "Gauge Fields and Strings", Contemporary Concepts in Physics, volume 3, Harwood Academic Publishers, Chur, 1987.

[2] I. Bakas, "Renormalization group flows and continual Lie algebras", JHEP $\underline{0308}$ (2003) 013.

[3] D. Friedan, "Nonlinear models in $2+\epsilon$ dimensions", Ann. Phys. 134 (1981) 85.

[4] H.-D. Cao and B. Chow, "Recent developments on the Ricci flow", Bull. Am. Math. Soc. $\underline{36}$ (1999) 59 .

[5] M. Saveliev and A.M. Vershik, "Continual analogs of contragradient Lie algebras (Lie algebras with a Cartan operator and nonlinear dynamical systems)", Commun. Math. Phys. 126 (1989) 367.

[6] V.A. Fateev, E. Onofri and Al.B. Zamolodchikov, "Integrable deformations of the O(3) sigma model. The sausage model", Nucl. Phys. B406 (1993) [FS] 521.

[7] A. Adams, J. Polchinski and E. Silverstein, "Don’t panic! Closed string tachyons in ALE space-times", JHEP $\underline{0110}$ (2001) 029.

[8] M. Gutperle, M. Headrick, S. Minwalla and V. Schomerus, "Space-time energy decreases under world-sheet RG flow", JHEP $\underline{0301}$ (2003) 073.

[9] R. Gregory and J. Harvey, "Space-time decay of cones at strong coupling", Class. Quant. Grav. 20 (2003) L231.

[10] C. Gundlach, "Critical phenomena in gravitational collapse", Phys. Rept. $\underline{376}$ (2003) 339.

[11] A.B. Zamolodchikov, "Irreversibility of the flux of the renormalization group in a 2D field theory", JETP Lett. $\underline{43}$ (1986) 730.

[12] G. Perelman, "The entropy formula for the Ricci flow and its geometric applications", math.DG/0211159. 